\title{
Accurate Quantitation of Dystrophin Protein in Human Skeletal Muscle Using Mass Spectrometry

\author{
Kristy J. Brown ${ }^{1,2 \#}$, Ramya Marathi" ${ }^{1 \#}$, Alyson A. Fiorillo' ${ }^{1}$, Eugene F. Ciccimaro ${ }^{2}$, Seema Sharma ${ }^{3}$, David S. Rowlands ${ }^{4}$, Sree Rayavarapu ${ }^{1,2}$,
} Kanneboyina Nagaraju ${ }^{1,2}$, Eric P. Hoffman ${ }^{1,2}$ and Yetrib Hathout ${ }^{1,2 *}$
}

${ }^{1}$ Children's National Medical Center, Center for Genetic Medicine Research, USA

${ }^{2}$ Department of Integrative Systems Biology, The George Washington University, 2300 Eye Street, N.W., Ross 605, Washington, D.C. USA

${ }^{3}$ ThermoFisher Scientific, Life Sciences Mass Spectrometry, USA

${ }^{4}$ School of Sport and Exercise, Massey University, Wellington, New Zealand

\#Contributed equally

\begin{abstract}
Quantitation of human dystrophin protein in muscle biopsies is a clinically relevant endpoint for both diagnosis and response to dystrophin-replacement therapies for dystrophinopathies. A robust and accurate assay would enable the use of dystrophin as a surrogate biomarker, particularly in exploratory Phase 2 trials. Currently available methods to quantitate dystrophin rely on immunoblot or immunohistochemistry methods that are not considered robust. Here we present a mass spectrometry based approach to accurately quantitate dystrophin protein in a total protein extract from human muscle biopsies. Our approach uses a combination of stable isotope labeled dystrophin as a spike-in standard, gel electrophoresis and high precision mass spectrometry to detect and quantitate multiple peptides of dystrophin within a complex protein mixture. The method was found highly reproducible and linear over a wide dynamic range, detecting as low as $5 \%$ of dystrophin relative to the normal amount in healthy individuals.
\end{abstract}

Keywords: Duchenne; Dystrophin; Quantitation; Mass spectrometry; Stable isotope

\section{Introduction}

Dystrophin is a relatively large $(427 \mathrm{kDa})$ multi-domain protein that plays an essential role in muscle fiber integrity and function. It is predominantly expressed in skeletal muscle but other isoforms have been found expressed in heart and brain [1]. Dystrophin is a fairly low abundance protein, accounting for approximately $0.002 \%$ of the total striated muscle protein [2]. Gene mutations resulting in the complete loss of dystrophin protein expression are the cause of Duchenne muscular dystrophy (DMD), the most common and severe form of muscular dystrophy [3]. Mutations resulting in the production of a truncated but partially functional dystrophin protein give rise to a milder dystrophinopathy termed Becker muscular dystrophy (BMD). In general, BMD patients have longer lives and less severe clinical presentations than DMD patients. This is mainly due to the presence of the partially functional dystrophin protein in BMD patients [4]. But, BMD patients do have a wide variety of phenotypes ranging from mild to severe most probably due the variable amounts of expressed dystrophin often seen in BMD population [5].

The need to accurately detect and quantitate dystrophin in human skeletal muscle biopsies is becoming crucial as new promising therapies aiming to restore dystrophin expression, especially in DMD patients, are entering phase II/III clinical trials [6-10]. Perhaps one of the most promising new generation therapies for DMD is 'exon skipping' which has been effective in restoring dystrophin expression and muscle force in both mouse and dog models of DMD [11,12]. The goal of this approach is to 'skip' an exon neighboring a deletion mutation during pre-mRNA splicing, thereby restoring the mRNA reading frame, resulting in the production of a shorter length, partially functional Becker-like dystrophin protein. Two pharmaceutical companies are currently in clinical trials with different exon skipping chemistries for the treatment of Duchenne muscular dystrophy $[6,10]$.

The most widely used clinical endpoint in Duchenne muscular dystrophy clinical trials is the 6 minute walk test [13]. However, significant changes in walking speed may take a year or more to develop, and there is significant test to test variation. Thus, relying on functional outcome measures such as the 6 minute walk increase the number of patients needed for adequate statistical powering, as well as the length of trial, and also increase the cost of conducting trials.

This is a particularly significant barrier to Phase 2 trials, where a more rapid read-out showing a proof-of-principle of the therapeutic approach is desired. Quantitation of dystrophin protein is an ideal endpoint measure [14] and clinically meaningful since it is directly linked to the disease. However, current methods for dystrophin quantitation such as western blots and immunohistochemistry are notoriously difficult and inconsistent. There have been successful attempts to improve immunohistochemistry quantitation, but it remains an indirect and fairly subjective method of quantitating dystrophin $[15,16]$. Furthermore, all antibody based approaches are limited due to dilution issues and inability to distinguish between splice isoforms. Alternatively, mRNA quantitation was also used as an assay to evaluate the levels of exon skipped products [17]. But, mRNA expression does not always correlate with protein levels [18] and amplification saturation can be problematic. Finally, both antibody based assays and mRNA approaches as surrogate biomarkers suffer from limited reliability and sensitivity. In an effort to overcome these hurdles, we have developed a quantitative dystrophin protein assay using high precision mass spectrometry in

*Corresponding author: Yetrib Hathout, Children's National Medical Center, Center for Genetic Medicine Research, 111 Michigan Avenue, NW, Washington, USA, Tel: 202-476-3136; Fax: 202-476-6014; E-mail: yhathout@cnmcresearch.org

Received November 27, 2012; Accepted December 13, 2012; Published December 18, 2012

Citation: Brown KJ, Marathi R, Fiorillo AA, Ciccimaro EF, Sharma S, et al. (2012) Accurate Quantitation of Dystrophin Protein in Human Skeletal Muscle Using Mass Spectrometry. J Bioanal Biomed S7: 001. doi:10.4172/1948-593X.S7-001

Copyright: @ 2012 Brown KJ, et al. This is an open-access article distributed under the terms of the Creative Commons Attribution License, which permits unrestricted use, distribution, and reproduction in any medium, provided the original author and source are credited. 
combination with stable isotope spike-in strategy. This technique detects and quantitates dystrophin protein in small amounts of total muscle extracts and is compatible with full length or truncated Beckerlike dystrophin proteins. This assay shows features of a robust surrogate biomarker sought for Phase 2 trials focused on dystrophin replacement in DMD.

\section{Methods}

\section{Muscle biopsies}

All human muscle biopsies used in this study were previously banked in our laboratory in accordance with an Institutional Review Board approved protocol at Children's National Medical Center (CNMC) and after obtaining patients written informed consent. Only excess specimens after diagnosis were used. Diagnosis was previously performed at the Center for Genetic Medicine at Children's National Medical Center. Banked specimens were deidentified and only age, gender, diagnosis and phenotype were reported to us. Total protein extracts of muscle biopsies from healthy subjects were obtained from Dr. David Rowlands at Massey University, Wellington, New Zealand.

\section{Custom peptides}

Custom dystrophin peptides LLDLLEGLTGQK, LLVEELPLR, IFLTEQPLEGLEK and filamin $c$ peptide VYNVTYTVK with ${ }^{13} \mathrm{C}_{6}$ ${ }^{15} \mathrm{~N}_{2}$-Lysine and ${ }^{13} \mathrm{C}_{6}{ }^{-15} \mathrm{~N}_{4}$ - Arginine were purchased from New England Peptide (Gardner, MA). Peptides were suspended in 50\% molecular grade acetonitrile containing $0.1 \%$ TFA and spiked into samples prior to mass spectrometric analyses.

SILAM/SILAC mouse: Custom "heavy" C57BL6 mice, termed SILAM or SILAC Mouse (stable isotopic labeling by amino acid in a mouse/mammal) were generated in-house and handled according to Institutional Animal Care and Use Committee guidelines at the Children's National Medical Center (Approved protocol \# 199-0701). Mice were fed a custom diet containing ${ }^{13} \mathrm{C}_{6}$-Lysine (Cambridge Isotope Laboratories, Andover, MA) at 1\%, adhering to laboratory mouse nutritional standards. A pregnant dam was fed with 'heavy' diet until a litter (F1 generation) was obtained and continued through weaning of the pups. After weaning, the pups were continued on 'heavy' diet through breeding and generation of a F2 litter. Label incorporation was monitored for specific organs and specific proteins by mass spectrometry. Labeling efficiency was better than $96 \%$ by F2 generation, including skeletal muscle [19]. This labeling efficiency was similar to labeling efficiencies obtained by others [20]. SILAC labeled skeletal muscles were harvested, flash frozen in liquid nitrogen chilled isopentane and stored at $-80^{\circ} \mathrm{C}$ until analysis as previously described [19].

\section{Muscle protein preparation}

Approximately 50 serial sections were prepared from each of the human muscle biopsies and SILAC mouse gastrocnemius muscle using a cryostat set at $10 \mu \mathrm{m}$ thickness. Muscle sections were directly placed into a microcentrifuge tube on dry ice. Total protein extraction from the muscle samples was performed using a handheld homogenizer either in a RIPA buffer (ThermoScientific) or a modified Laemmli sample buffer containing 10\% SDS (10\% SDS, 75 mM Tris- $\mathrm{HCl}$, pH 6.8, 10 mM EDTA, 20\% glycerol, 50 mM DTT) [21]. Protein concentration was determined using a BCA assay (Pierce, Thermo Scientific).

\section{Dystrophin quantitation using mass spectrometry with stable isotope spike-in strategy}

Two approaches were evaluated to quantitate dystrophin in skeletal muscle extracts (Figure 1). In the first approach, called post-digestion spike-in approach hereafter, aliquots of $50 \mu \mathrm{g}$ total protein from each muscle extract was diluted in LDS buffer (Invitrogen, Carlsbad, CA) and further fractionated by SDS-PAGE on a 3-8\% Tris-Acetate midi gel (Invitrogen) for 1 hour at $160 \mathrm{~V}$. Gels were stained with BioSafe commassie (BioRad) for 1 hour. The upper section of the gel (300 to $450 \mathrm{kDa}$ ) containing dystrophin protein and other skeletal muscle proteins was excised and processed for in-gel digestion as previously described [22] using proteomics grade trypsin (Promega, Madison, WI). Peptides were extracted from the gel and spiked with known amounts (14 nM, $40 \mathrm{nM}, 80 \mathrm{nM}$ and $160 \mathrm{nM}$ ) of custom synthesized stable isotope labeled dystrophin peptides as well as an internal control filamin c peptide. In the second approach, called pre- digestion spikein approach hereafter, aliquots of $50 \mu \mathrm{g}$ of total protein from each muscle extract were spiked with $25 \mu \mathrm{g}$ of muscle extract prepared from ${ }^{13} \mathrm{C}_{6}$-Lysine labeled SILAC mouse. The mixture was then fractionated by SDS-PAGE on a 3-8\% Tris-Acetate gel and gel bands in the 300$450 \mathrm{kDa}$ were processed as above. To determine the limit of detection (LOD) and limit of quantitation (LOQ) of dystrophin protein in a complex muscle extract we used a muscle extract from a DMD patient, completely lacking dystrophin protein, and mixed at different ratios with a muscle extract from a healthy donor that expresses dystrophin as follows: DMD to normal $100 \% / 0 \%, 95 \% / 5 \%, 90 \% / 10 \%, 75 \% / 25 \%$, $50 \% / 50 \%, 25 \% / 75 \%, 10 \% / 90 \%$, 5\%/95\%, $0 \% / 100 \%$. The final protein content in each mixture was kept constant at $50 \mu \mathrm{g}$. Each sample was then spiked with $25 \mu \mathrm{g}$ of muscle extract prepared from ${ }^{13} \mathrm{C}_{6}$-Lys labeled SILAM mouse to quantitate human dystrophin. Samples were analyzed using two different types of mass spectrometry instruments. For analysis by LTQ-Orbitrap-XL (Thermo), concentrated peptides from each spiked sample above were dissolved in $10 \mu \mathrm{L}$ of $0.1 \%$ TFA solution and $6 \mu \mathrm{L}$ was injected via an autosampler onto a Symmetry C18 trap column $(5 \mu \mathrm{m}, 300 \mu \mathrm{m}$ i.d., $23 \mathrm{~mm}$, Waters) for $10 \mathrm{~min}$ at a flow rate of $10 \mu \mathrm{m} / \mathrm{min}, 100 \% \mathrm{~A}$. The sample was subsequently separated by a C18 reverse-phase column $(3 \mu \mathrm{m}, 200 \mathrm{~A}, 100 \mu \mathrm{m} \times 15 \mathrm{~cm}$, Magic C18, Michrom Bioresources) at a flow rate of $330 \mathrm{~nL} / \mathrm{min}$ using an Eksigent nano-hplc system (Dublin, CA). The mobile phases consisted of water with $0.1 \%$ formic acid (A) and $90 \%$ acetonitrile (B). A 65 minute linear gradient from 5 to $40 \%$ B was employed. Eluted peptides were introduced into the mass spectrometer via a Michrom Bioresources CaptiveSpray. The spray voltage was set at $1.4 \mathrm{kV}$ and the heated capillary at $200^{\circ} \mathrm{C}$. The LTQ-Orbitrap-XL was operated in data-dependent mode with dynamic exclusion in which one cycle of experiments consisted of a full-MS in the Orbitrap (300-2000 m/z) survey scan in profile mode, resolution 30,000, and five subsequent MS/MS scans in the LTQ of the most intense peaks in centroid mode using collision-induced dissociation with the collision gas (helium) and normalized collision energy value set at $35 \%$.

For Q Exactive analysis, concentrated peptides from each spiked sample above were redissoleved in $20 \mu \mathrm{L}$ of 90:10 water:acetonitrile with $0.1 \%$ TFA containing $2.5 \mathrm{fmol} / \mu \mathrm{L}$ of trainer peptide mixture. Sample were then injected via autosampler followed by separation on a HALO C18 column $(75 \mu \mathrm{m}$ ID, $10 \mathrm{~cm}, 2.7 \mu \mathrm{m})$ with a gradient of 0 to $35 \%$ (A: $0.1 \%$ formic acid water, B: $0.1 \%$ formic acid acetonitrile) at a flow rate of $400 \mathrm{~nL} / \mathrm{min}$ on a Thermo Scientific Easy 1000 connected to 
Citation: Brown KJ, Marathi R, Fiorillo AA, Ciccimaro EF, Sharma S, et al. (2012) Accurate Quantitation of Dystrophin Protein in Human Skeletal Muscle Using Mass Spectrometry. J Bioanal Biomed S7: 001. doi:10.4172/1948-593X.S7-001

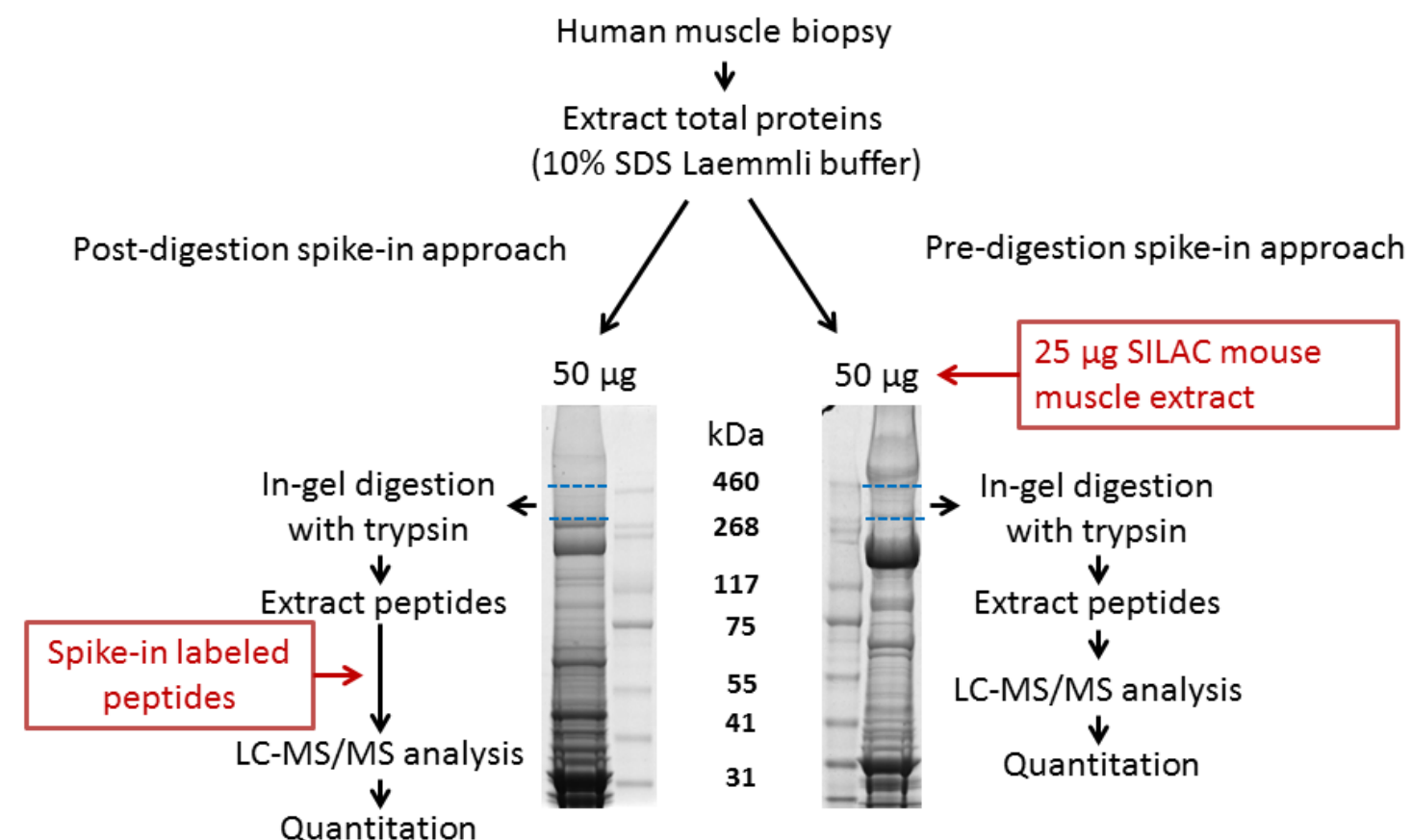

Figure 1: Overview of the experimental design used to quantitate dystrophin protein in human skeletal muscle.

Total protein extracts from muscle biopsies (50 $\mu \mathrm{g}$ aliquots) were further fractionated on SDS-PAGE using 3-8\% Tris-Acetate gel, which is suitable for high molecular mass proteins. Left panel shows the post-digestion spike-in approach where the area corresponding to dystrophin migration level (dashed blue lines) was serially sliced, in-gel digested by trypsin and spiked with stable isotope labeled standard peptides (see supplemental Figure S2 for peptide sequences and their location within the dystrophin protein) for subsequent LC-MS/MS analysis and peptide quantitation. In the Pre-digestion spike-in approach (right panel), $50 \mu \mathrm{g}$ total muscle protein extract was spiked with $25 \mu \mathrm{g}$ of ${ }^{13} \mathrm{C}_{6}$-Lysine labeled SILAC mouse muscle protein extract. The mixture was then fractionated by SDS-PAGE and the area corresponding to dystrophin migration was excised, in-gel digested and the resulting peptides were analyzed by LC-MS/MS for identification and quantitation.

a nanoESI flex source. For the peptide coverage discovery experiment, the Q Exactive was operated with a full scan $(380-1500 \mathrm{~m} / \mathrm{z})$ at 70,000 resolution followed by MS/MS of the top ten most abundant ions at 17,500 resolution. For quantitative acquisition, the Q Exactive was operated with a full scan $(380-1500 \mathrm{~m} / \mathrm{z})$ at 35,000 resolution followed by timed targeted MS/MS of dystrophin and internal stable isotope labeled standard peptides at 35,000 resolution.

\section{Protein identification and quantitation}

LTQ-Orbitrap XL Raw files were searched using Sequest in the Bioworks Browser (Thermo) software against a tryptic human database with 2 missed cleavages, $50 \mathrm{ppm}$ mass accuracy, $1.5 \mathrm{Da}$ fragment mass tolerance. Spiked peptides were identified and elution times documented. Dystrophin was quantitated by comparing the unlabeled to the spiked-in heavy isotope profile for each peptide. Q Exactive Raw files were searched with Proteome Discoverer 1.3 (ThermoFisher Scientific) using SEQUEST and the human refseq. database (release 47, ftp://ftp.ncbi.nih.gov/refseq/H_sapiens/mRNA_Prot/, with 34,340 entries). Search parameters allowed trypsin to cleave after Lysine and Arginine with 2 missed cleavages. Precursor ion mass tolerance was set to $10 \mathrm{ppm}$ and fragment ion mass tolerance was $20 \mathrm{ppm}$. The dynamic modification of methionine (oxidation $=15.995 \mathrm{Da}$ ) and lysine $\left({ }^{13} \mathrm{C}_{6}\right.$ $=6.020 \mathrm{Da}$ ), was accepted on up to 4 residues per peptide. Within the Proteome Discoverer Software, SILAC pairs were identified using the "2 plex" workflow node, and all peptides were rescored using the Percolator algorithm node. Finally, peptides were filtered at $1 \%$ FDR. SILAC quantification from $25 \%$ and $75 \%$ normal were compared and proteins showing a consistent ratio were considered as controls. Peak areas and area ratios were calculated with Pinpoint software using recreated ion chromatograms at $25 \mathrm{ppm}$ from the full scan MS/MS. The ratios of the integrated areas for endogenous peptides and $\left[{ }^{13} \mathrm{C}_{6}\right.$ lys] labeled peptides were calculated to obtain peptide measures using multiple y-ion fragments per peptide (Supplementary Figure S1). The average of these peptide ratios determined the relative amount of each protein.

\section{Results}

High SDS containing buffer enabled efficient dystrophin extraction from skeletal muscle biopsies

Dystrophin protein is a low abundant protein, accounting for only $0.002 \%$ of total muscle protein. Since no amplification technologies exist for protein detection by mass spectrometry, efficient dystrophin extraction was essential. In addition to efficient extraction, enrichment of dystrophin protein from other more abundant muscle proteins prior to mass spectrometry was required in order to reduce the sample dynamic range and thereby ensure consistent detection of dystrophin. We evaluated a mild (RIPA buffer) and strong (10\% SDS) buffer for muscle protein extraction. As expected the $10 \%$ SDS modified Laemmli buffer extracted 10 times more total protein than the RIPA buffer from the same amount of muscle tissue. Indeed, about $8 \mu \mathrm{g}$ and $54 \mu \mathrm{g}$ of total proteins were extracted from $1 \mathrm{mg}$ of fresh muscle tissue using RIPA and $10 \%$ SDS modified Laemmli buffer, respectively. The increased extraction efficiency of the SDS buffer also increased the dynamic range of the sample by extracting more of the highly abundant skeletal muscle proteins such as myosins, titin and nebulin. To overcome the dynamic range limitations, total muscle extract from each of the RIPA 
Citation: Brown KJ, Marathi R, Fiorillo AA, Ciccimaro EF, Sharma S, et al. (2012) Accurate Quantitation of Dystrophin Protein in Human Skeletal Muscle Using Mass Spectrometry. J Bioanal Biomed S7: 001. doi:10.4172/1948-593X.S7-001

and SDS preparation was further separated on a 3-8\% Tris-Acetate gel. The region on the gel corresponding to the migration zone of dystrophin and/or truncated Becker-like dystrophin (300-450 kDa) was excised, and processed for mass spectrometry analysis as described above. Dystrophin protein was identified by 19 and 50 peptides when muscle sample was extracted with RIPA and $10 \%$ SDS modified Laemmli buffers, respectively (Supplementary Table S1). The location of these peptides throughout the dystrophin sequence is shown in Supplementary Figure S2.

\section{Dystrophin quantitation by mass spectrometry}

We evaluated two approaches to quantitate dystrophin protein in total muscle protein extracts (Figure 1): (i) Post-Digestion spikein approach with stable isotope labeled custom synthesized standard dystrophin peptides; and (ii) Pre-Digestion spike-in approach with stable isotope labeled mouse muscle protein extract prior to gel separation and in-gel digestion.

\section{Post-digestion spike-in approach}

For the post-digestion spike-in approach, three stable isotope labeled dystrophin peptides were custom synthesized. The sequences of these peptides were chosen based on their consistent and reproducible detection by LC-MS/MS following in-gel digestion of SDS-PAGE fractionated muscle extracts, different retention times, amino acid content (e.g. absence of highly modifiable residues such as cysteine and methionine), as well as the location within the dystrophin sequence (e.g. distributed throughout the $\mathrm{N}$-terminal, middle and C-terminal domain). Additionally, a stable isotope labeled peptide for filamin $c$ was also custom synthesized and used as an internal standard. The sequences of the standard peptides, their retention time, observed molecular masses as well as their location within the dystrophin sequence are highlighted in Supplementary Figure S3. First the postdigestion spike-in approach was optimized for the amount of stable isotope labeled standard peptides to be used for spike-in. In-gel digested dystrophin containing bands were prepared from replicate SDS-PAGE fractionation of $50 \mu \mathrm{g}$ of muscle protein extracts of a healthy donor and spiked with varying amounts of stable isotope labeled standard peptides (14 nM, $40 \mathrm{nM}, 80 \mathrm{nM}$ and $160 \mathrm{nM}$ ). Figure 2 shows MS spectra of the unlabeled peptide from the endogenous dystrophin and its corresponding heavy stable isotope spiked-in standard at varying concentrations. From this preliminary study the optimal amount of stable isotope labeled peptides to spike-in ranged between 14 and 40 $\mathrm{nM}$. Spiking amounts below $14 \mathrm{nM}$ resulted in poor signal to noise spectra while spiking amounts above $40 \mathrm{nM}$ resulted in increased
(A)
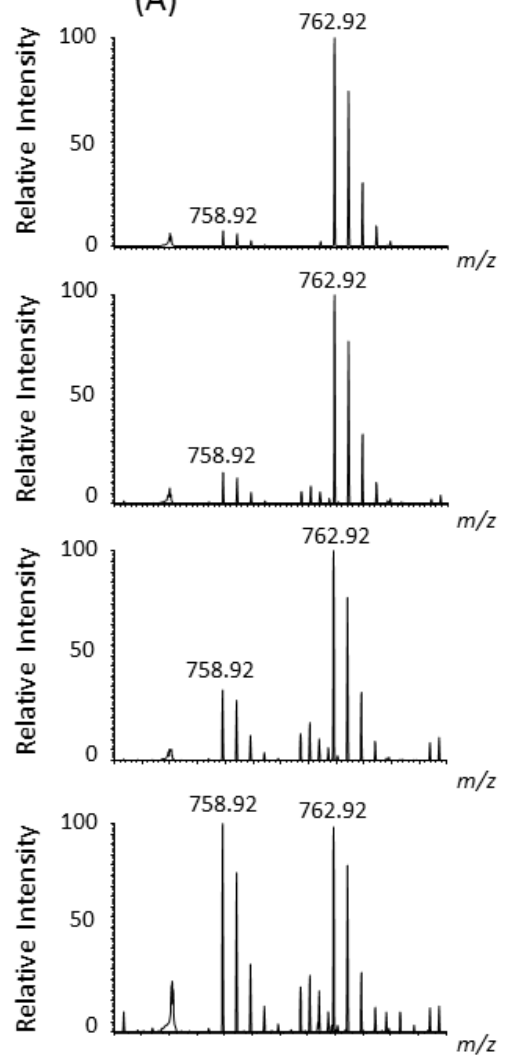

(B)
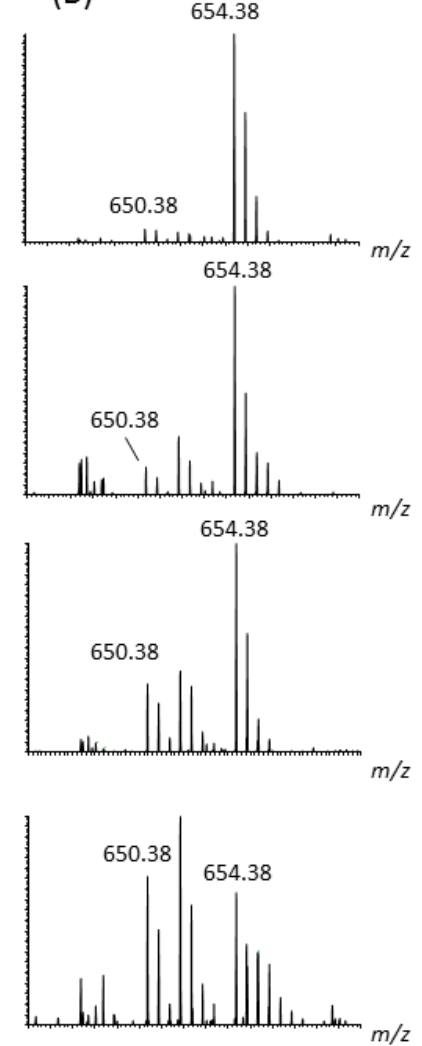

(C)
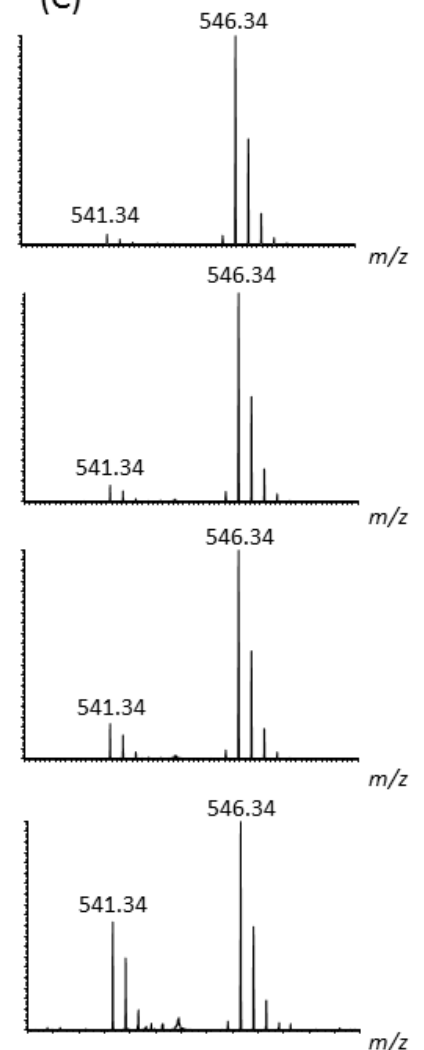

Figure 2: Representative mass spectra of spiked-in labeled standard peptides and corresponding unlabeled endogenous peptide using post-digestion spike-in approach. (A) Mass spectra of labeled and unlabeled peptide pairs detected after spiking in-gel digested dystrophin with varying amounts of ${ }^{13} \mathrm{C},{ }^{15} \mathrm{~N}_{2}$ Lysine labeled standard peptide [IFLTEQPLEGLEK]. (B) Shows mass spectra of labeled and unlabeled peptide pairs detected after spiking in-gel digested dystrophin with varying amounts of ${ }^{13} \mathrm{C}_{6},{ }^{15} \mathrm{~N}_{2}$ Lysine labeled standard peptide [LLDLLEGLTGQK], (C) Shows mass spectra of labeled and unlabeled peptide pairs detected after spiking in-gel digested dystrophin with varying amounts of ${ }^{13} \mathrm{C}_{6},{ }^{15} \mathrm{~N}_{4}$ Lysine labeled standard [LLVEELPLR]. The concentration of each spike-in standard peptide, starting for the top to the low panels, was as follows (160 nM, $80 \mathrm{nM}, 40 \mathrm{nM}$ and $14 \mathrm{nM})$. Samples were analyzed on the LTQ-Orbitrap XL at 30,000 resolution and mass error better than 10 $\mathrm{ppm}$. All ions were detected as doubly protonated species with the correct $\mathrm{m} / \mathrm{z}$ values. 
Citation: Brown KJ, Marathi R, Fiorillo AA, Ciccimaro EF, Sharma S, et al. (2012) Accurate Quantitation of Dystrophin Protein in Human Skeletal Muscle Using Mass Spectrometry. J Bioanal Biomed S7: 001. doi:10.4172/1948-593X.S7-001

dynamic range between the standard peptide and the endogenous peptide ion intensities reducing the linear range for quantitation. To test the reproducibility of the post-digestion spike-in approach, triplicate extraction experiments performed on the same muscle biopsy obtained from a healthy donor were processed and spiked with an optimal amount of $30 \mathrm{nM}$ of stable isotope labeled standard peptide mixture. Figure 3 shows the reproducibility of the triplicate assay with a CV of less than $7 \%$. Unfortunately, the reproducibility of the experiment did not hold across the three different dystrophin targeted peptides for a titration curve of endogenous dystrophin protein including the internal control protein filamin c (Figure 4). This was attributed to the inherent variability of in-gel digestion. Therefore, the post-digestion spike-in approach suffered from high sample handling errors.

\section{Pre-digestion spike-in approach}

In order to overcome in-gel digestion variability we evaluated a pre-digestion spike-in approach using ${ }^{13} \mathrm{C}_{6}$-Lysine fully labeled dystrophin protein obtained from muscle extracts prepared from a SILAC mouse. In this method the sample is spiked at the protein level prior to electrophoresis and in-gel digestion, thus minimizing variation due to sample handling and greatly improving reproducibility.
Since production of a fully labeled human dystrophin protein using transfection technology and over-expression in a labeled culture system was challenging due to the low transfection rate of the largest human gene [23], use of ${ }^{13} \mathrm{C}_{6}$-Lysine labeled dystrophin prepared from a SILAC mouse was employed [20,24]. Mouse dystrophin and human dystrophin have 91\% sequence homology (Supplementary Figure S4). Our lab had previously generated a fully ${ }^{13} \mathrm{C}_{6}$-Lysine labeled SILAC mouse [19]. Skeletal muscle from the SILAC mouse was sectioned by cryostat, total protein containing $0.002 \%$ of fully labeled dystrophin were extracted using the $10 \%$ SDS modified Laemmli buffer then used as a spike-in standard in human muscle extracts. Spiked human muscle extracts were further fractionated by SDS-PAGE and processed for mass spectrometry analysis using the Q Exactive instrument as described above. In a discovery run, 43 unique dystrophin peptides were detected with good coverage throughout the entire dystrophin sequence. Of these 43 unique peptides, five terminated in lysine and had 100\% sequence homology with mouse tryptic peptides and therefore were used to quantitate dystrophin in human muscle extracts (Supplementary Figure S4). In addition to these dystrophin overlapping peptides between human and mouse, other lysine terminating peptides belonging to several other skeletal muscle proteins also had $100 \%$

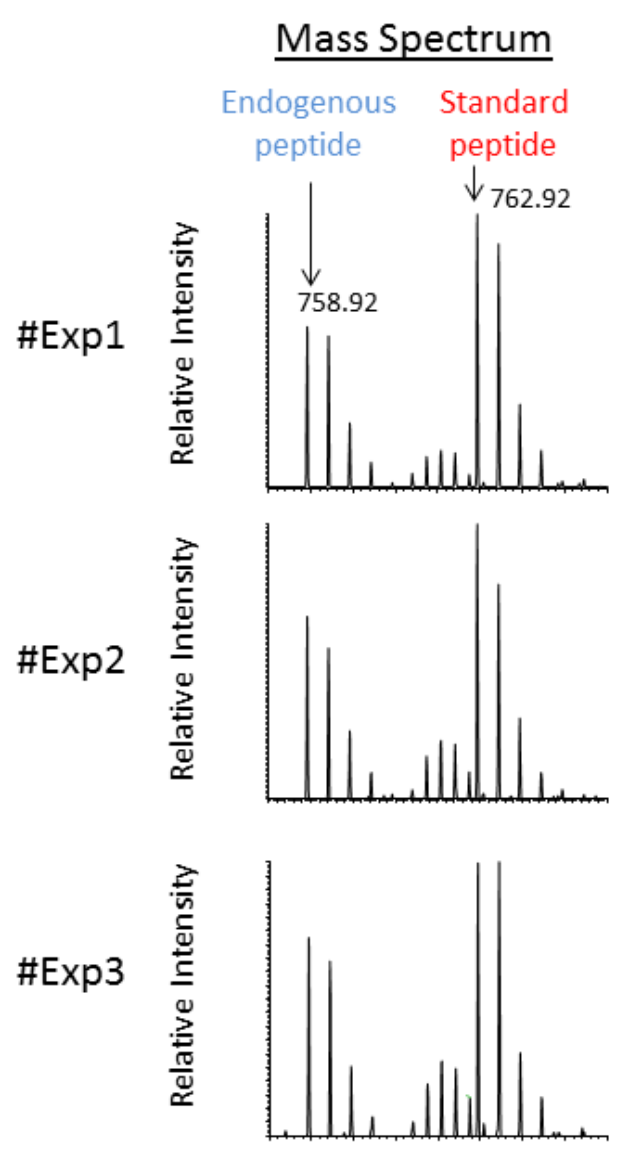

\section{Extracted Ion Chromatogram}
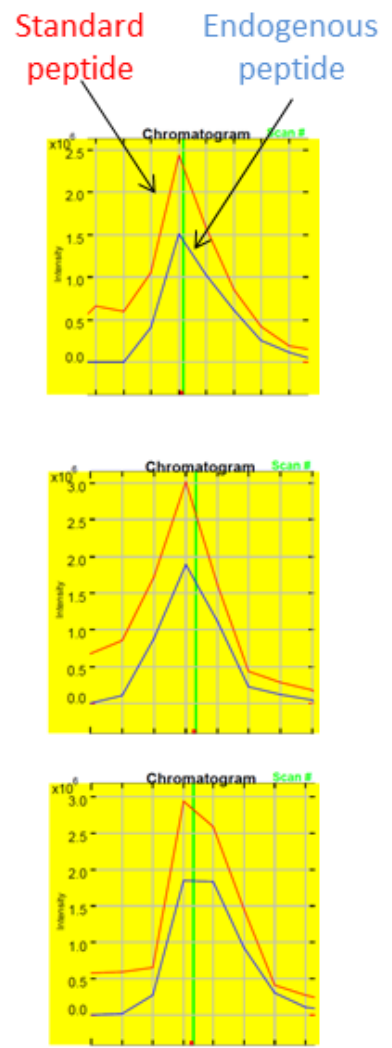

Figure 3: Technical reproducibility of the post-digestion spike-in approach showing mass spectrometry data obtained for triplicate experiments using the same extract from a muscle biopsy obtained from a healthy donor.

Three aliquots of $50 \mu \mathrm{g}$ total muscle protein extract were separated by SDS-PAGE as described in Methods. The migration area corresponding to dystrophin was excised, in-gel digested and spiked with optimal concentration (30 nM) of stable isotope labeled standard peptides. The left panel shows MS spectra of unlabeled peptide [IFLTEQPLEGLEK] generated from endogenous dystrophin and the spike-in stable isotope labeled standard peptide [IFLTEQPLEGLEK]. Both peptides were detected as doubly charged ions at their corresponding $\mathrm{m} / \mathrm{z}$ value of 758.92 and 762.92 , respectively. The right panel shows extracted ion chromatograms used for ratio measurement of unlabeled endogenous dystrophin peptide (blue trace) to the stable isotope labeled standard peptide (red trace). 
sequence homology between human and mouse. This enabled the evaluation and selection of appropriate internal standards for accurate dystrophin quantitation (next section).

\section{Limit of detection and limit of quantitation of dystrophin protein in total muscle extract}

To evaluate the LOD and LOQ of the pre-digestion spike-in approach, we used targeted mass spectrometry analysis on the five dystrophin peptides detected above as well as a few internal control peptides from myomesin and filamin c. Dystrophin protein was targeted for quantitation in the following combination of normal and DMD muscle extracts: $0 \% / 100 \%, 5 \% / 95 \%, 10 \% / 90 \%, 25 / 75 \%$, $50 / 50 \%, 75 / 25 \%, 90 / 10 \%, 95 / 5 \%, 100 / 0 \%$. Each mixture had a final protein content of $50 \mu \mathrm{g}$. The range of dystrophin protein expression, from $0 \%$ to $100 \%$ of normal, is thus artificially generated to represent possible amounts of dystrophin protein in Becker patients or in Duchenne patients receiving treatments aiming to restore dystrophin expression [6-10]. Each combination mixture above $(50 \mu \mathrm{g}$ final total protein) was then spiked with SILAC ${ }^{13} \mathrm{C}_{6}$-Lysine labeled SILAC mouse muscle extract $(25 \mu \mathrm{g})$. Samples were fractionated on a $3-8 \%$ Tris-Acetate gel, bands in the dystrophin protein region (300-450 $\mathrm{kDa}$ ) were excised, in-gel digested and analyzed by LC-MS/MS on a Q Exactive mass spectrometer using a timed targeted MS/MS method. Unlabeled and labeled dystrophin, filmain $\mathrm{c}$ and myomesin peptides from both the human muscle extract and the spike-in SILAC mouse labeled extract were targeted for MS/MS analysis. Selected transition ions were used for quantitation in each sample. The average ratio of labeled to unlabeled peptide intensities for dystrophin, filamin $\mathrm{c}$ and myomesin were plotted against the different muscle combination mixtures resulting in a calibration curve with an $\mathrm{R}^{2}$ of 0.99 (Figure 5). While the amount of dystrophin increased with increasing \% of normal muscle extract in the mixtures, two muscle specific proteins, filamin $\mathrm{c}$ and myomesin, remained relatively unchanged. These two proteins are therefore ideal candidates as internal controls. Note that filamin $\mathrm{c}$ and myomesin did show a slight increase in abundance as the $\%$ of normal increased (Figure 5), and this is expected since the amount of muscle present in dystrophic tissue is slightly decreased and is replaced by fibrotic tissue (e.g. hallmark of DMD pathogenesis) [25]. Therefore, monitoring of internal standards across the curve allows for improved accuracy and normalization.

\section{Absolute quantitation of dystrophin in becker patients}

The absolute amount of dystrophin in muscle biopsies from normal donors $(n=12)$ and Becker patient donors $(n=5)$ was determined. Dystrophin in normal muscle ranged from 0.001 to $0.004 \%$ of total striated muscle, consistent with previous findings [2] The Becker samples also showed a wide range of dystrophin expression at approximately $10 \%$ of the level found in normal muscle (Figure 6). Dystrophin protein represents approximately $0.002 \%$ of total striated muscle protein and since the amount of spike-in standard is known, we estimated the total amount of dystrophin in healthy individuals to be $75 \pm 15 \mathrm{ng}$ per mg of muscle while in Becker patients the amount can range from 0 to $7 \mathrm{ng}$ per mg of muscle (Figure 6).

\section{Discussion}

In skeletal muscle, dystrophin protein is located just beneath the sarcolemma connecting the cytoskeleton to the extracellular matrix via a dystrophin associated glycoprotein complex (DAC or DGC) and thus stabilizes the sarcolemma of the muscle fiber and protects it from damage during the repeated cycles of contraction and relaxation.

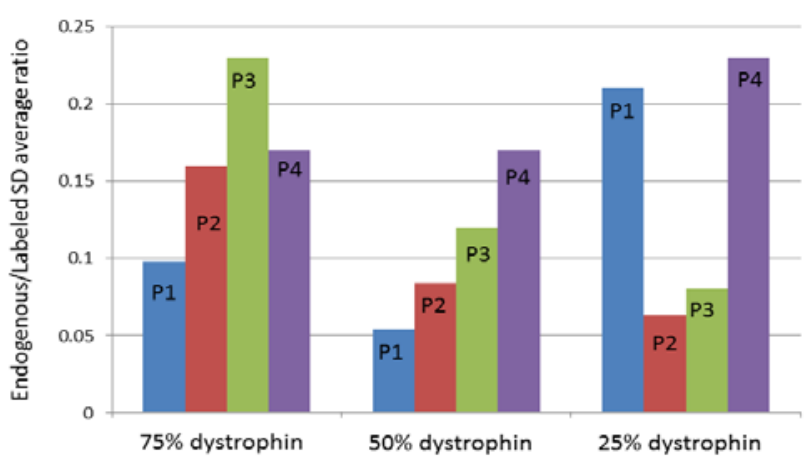

Figure 4: Inter sample reproducibility of the post-digestion spike-in approach. Three samples containing varying amount of dystrophin obtained by mixing muscle extract from a DMD and age matched healthy donor at ratio of 25/75, $50 / 50$ and $75 / 25$ respectively were evaluated for dystrophin quantitation using the post-digestion spike-in approach with $30 \mathrm{nM}$ of stable isotope standard peptides. Histograms represent intensity ratios of endogenous dystrophin peptides and filamin c peptide to their corresponding stable isotope labeled standard peptide. P1, P2 and P3 corresponds to dystrophin peptides [LLDLLEGLTGQK] [LLVEELPLR], [IFLTEQPLEGLEK] respectively and P4 correspond to filamin $c$ peptide [VYNVTYTVK] that was used as an internal control. It is clearly seen that some ratios did not decrease with decreasing amounts of dystrophin, especially for the peptide $\mathrm{P} 1$, showing high variability for this approach.

Mutations in the dystrophin gene, resulting in decreased or lack of dystrophin expression, such as in BMD and DMD patients, results in destabilization of the DAC complex and muscle fiber degeneration.

A number of promising treatment strategies aiming to restore dystrophin expression in DMD patients are in clinical development. These include exon skipping with antisense oligonucleotide $[6,10]$, stop codon read-through with Ataluren (PTC124) drug [7], gene therapy [26] and stem cell therapy [27]. One of the outcome measures to evaluate efficacy of these novel treatments requires accurate quantitation of restored dystrophin in muscle biopsies. Current antibody-based dystrophin assays, including both western blot and immunohistochemistry, are an indirect means by which to quantitate dystrophin, and are not considered highly reliable. Here we evaluated a promising mass spectrometry assay for accurate dystrophin quantitation in muscle. The technique uses a stable isotope labeled spike-in standard followed by high precision mass spectrometry analysis. In this study we found that spiking the muscle extracts with full length labeled muscle proteins prior to gel fractionation and in-gel digestion to be reliable and sensitive, and preferred to postdigestion peptide spike-in approach. Indeed, in-gel digestion variation rendered the latter approach less reproducible resulting in fluctuation of dystrophin measurement from run to run. Additionally, spiking samples with fully labeled muscle extracts enabled the use of other muscle specific proteins (filamin $\mathrm{c}$ and myomesin) as internal controls. Muscle biopsies, from normal or from patients with dystrophinopathies, are very heterogeneous containing varying proportions of myofibers, connective tissue (fibrosis) and adipose tissue (fatty replacement). Depending on the biopsy location and disease state, the proportion of myofibers (cell type expressing dystrophin) can vary significantly. Therefore, measuring myofiberspecific proteins to use as internal controls will allow normalization and precise quantitation of dystrophin protein in myofibers, and this is preferable to normalizing to total protein content of a biopsy. This was demonstrated in this study 
Citation: Brown KJ, Marathi R, Fiorillo AA, Ciccimaro EF, Sharma S, et al. (2012) Accurate Quantitation of Dystrophin Protein in Human Skeletal Muscle Using Mass Spectrometry. J Bioanal Biomed S7: 001. doi:10.4172/1948-593X.S7-001

by combining DMD and normal muscle extracts at different ratios. This strategy enabled a constant protein background while the amount of dystrophin changed.

In this assay the LOD and LOQ was better than $10 \%$ (e.g. as low as $5 \%$ ) of the normal amount of dystrophin, making the assay sensitive enough to measure dystrophin in clinical specimens. In this study we routinely measured dystrophin content in $50 \mu \mathrm{g}$ total muscle protein extract from roughly $0.5 \mathrm{mg}$ of frozen biopsy. This amount of muscle is readily available from diagnostic needle biopsies, which are in the range of $20 \mathrm{mg}$ [28].

Despite a sequence length of 3685 amino acids, only 45 to 50 dystrophin peptides were detected by mass spectrometry analysis of $50 \mu \mathrm{g}$ of total muscle protein extract. Increasing the amount of total muscle protein extract to $100 \mu \mathrm{g}$ did not improve dystrophin sequence coverage. This is mainly due to the inherent low abundance of this protein in muscle (e.g. dystrophin represents only about $0.002 \%$ of total striated muscle protein) and the presence of highly abundant proteins such as the myosins, which represent up to $40 \%$ of total muscle protein. Therefore it was necessary to pre-fractionation total protein muscle extract by SDS-PAGE to isolate dystrophin from highly abundant proteins and facilitate detection and quantitation in a complex biological matrix.

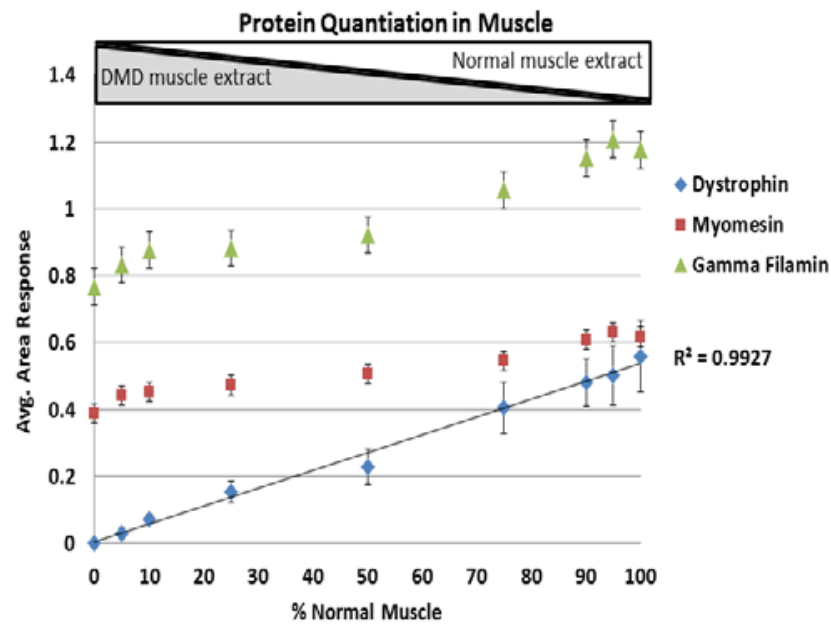

Figure 5: Titration curve of dystrophin protein and other muscle specific proteins using pre-digestion spike-in approach

Dystrophin protein, as well as myomesin and filamin c, were quantitated in different combination mixtures of muscle extracts from DMD and age matched healthy donors at ratios leading to $0,5,10,25,50,75,90,95$ and $100 \%$ of dystrophin relative to normal. Each final mixture contained $50 \mu \mathrm{g}$ of total muscle protein and was spiked with $25 \mu \mathrm{g}$ of ${ }^{13} \mathrm{C}_{6}$-Lysine labeled SILAC mouse muscle extract. Gel bands encompassing dystrophin protein and other muscle specific proteins were excised and processed for mass spectrometry analysis as described in Methods. Transition ion intensities obtained at the MS/MS level fo the targeted labeled and unlabeled peptide pairs generated from the spike-in standard and endogenous human dystrophin were used to determine the relative amount of dystrophin and other muscle proteins (myomesin and filamin c). Standard deviation at each data point represents average ratios obtained from labeled and unlabeled peptide pairs: 5 pairs for dystrophin protein (QAPIGGDFPAVQK, VLSQIDVAQK, IFLTEQPLEGLEK, TLNATGEEIIQQSSK, VHALNNVNK); 3 pairs for filamin c (SPFVVNVAPPLDLSK, EVGEHVVSVRK HIGISFTPK) and two pairs for myomesin (VSEPVAALDPAEK, VLGGLPDWTIQEGK). These peptides have $100 \%$ sequence homology between human and mouse and were used for ratio measurements. As expected, dystrophin protein increased with increasing relative amount of normal muscle extract while myomesin and filamin c remained unchanged.

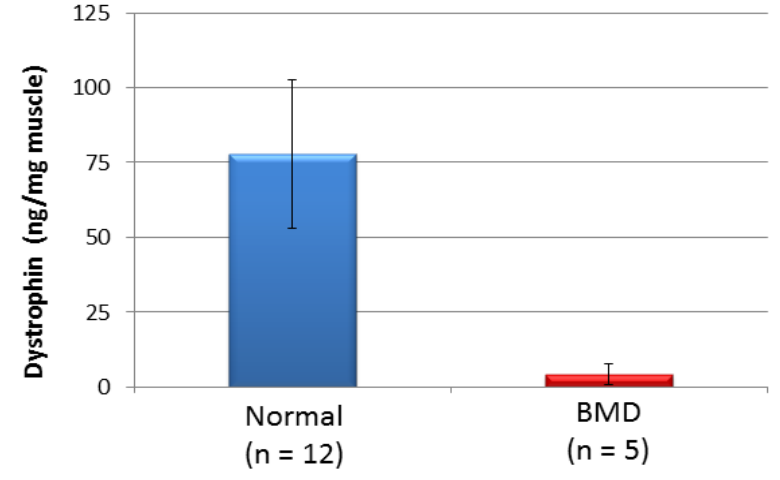

Figure 6: Calculated absolute amount of dystrophin in muscle biopsies obtained from BMD and age matched healthy controls.

Total protein extract $(50 \mu \mathrm{g})$ from each biopsy was spiked with $25 \mu \mathrm{g}$ of C -Lysine labeled SILAC mouse muscle extract and processed using the pre- digestion spike-in approach (see Figure 1 and Methods). Intensity ratios of labeled and unlabeled peptide pairs were used to determine the absolute amount of dystrophin in each muscle extract.

\section{Conclusions}

Targeted mass spectrometry quantitation of full length or truncated Becker dystrophin protein was facilitated by stable isotope spike-in strategy using full length ${ }^{13} \mathrm{C}_{6}$-Lysine labeled SILAC mouse muscle extract. The method was found to be highly reproducible, accurate and sensitive over a large dynamic range. Our aim is to apply this technique to quantitate restored dystrophin in DMD patients receiving exon skipping therapy to aid in the clinical evaluation of this promising new therapy. In the future, this technique could be refined to include quantitation of DAC complex proteins.

\section{Acknowledgements}

This work was partially supported by NIAMS 5P50AR060836-02 (Center for Research Translation of Systemic Exon-Skipping in Muscular Dystrophy), NIAMS R01AR062380 (Craig McDonald, PI), Massey University Research Fund, Wellington Medical Research Foundation Inc. and core grants NICHD/NINDS 2R24HD050846-06 (National Center for Medical Rehabilitation Research), NICHD 5P30HD040677-10 (Intellectual and Developmental Disabilities Research Center) and NIH NCATS UL1RR031988 (CTSI-CN). The authors would like to thank the following for their kind assistance: Dr. Jyoti Jaiswal, Dr. D. Ashley Hill, Dr. Mende Tuchman and Dr. Terrence Partridge for assistance in generating the SILAM SILAC mouse model and Dr. Akanchha Kesari for assistance in biobanking and retrieval of DMD and BMB biopsies.

\section{References}

1. Lederfein D, Levy Z, Augier N, Mornet D, Morris G, et al. (1992) A 71-kilodalton protein is a major product of the Duchenne muscular dystrophy gene in brain and other nonmuscle tissues. Proc Natl Acad Sci 89: 5346-5350.

2. Hoffman EP, Brown RH Jr, Kunkel LM (1987) Dystrophin: the protein product of the Duchenne muscular dystrophy locus. Cell 51: 919-928.

3. van Essen AJ, Busch HFM, te Meerman GJ, ten Kate LP (1992) Birth and population prevalence of Duchenne muscular dystrophy in The Netherlands. Hum Genet 88: 258-266.

4. Bushby KMD, Gardner-Medwin D, Nicholson LVB, Johnson MA, Haggerty ID, et al. (1993) The clinical, genetic and dystrophin characteristics of Becker muscular dystrophy. J Neurol 240: 105-112.

5. Anthony K, Sebahattin C, Silvia T, Giorgio T, Lucy F, et al. (2011) Dystrophin quantification and clinical correlations in Becker muscular dystrophy: implications for clinical trials. Brain 134: 3547-3559.

6. Sebahattin C, Virginia A, Michela G, Lucy F, Silvia T, et al. (2011) Exon skipping and dystrophin restoration in patients with Duchenne muscular dystrophy after systemic phosphorodiamidate morpholino oligomer treatment: an open-label phase 2, dose- escalation study. Lancet 378: 595-605. 
Citation: Brown KJ, Marathi R, Fiorillo AA, Ciccimaro EF, Sharma S, et al. (2012) Accurate Quantitation of Dystrophin Protein in Human Skeletal Muscle Using Mass Spectrometry. J Bioanal Biomed S7: 001. doi:10.4172/1948-593X.S7-001

7. Finkel RS (2010) Read-through strategies for suppression of nonsense mutations in Duchenne/ Becker muscular dystrophy: aminoglycosides and ataluren (PTC124). J Child Neurol 25: 1158-1164.

8. Nelson SF, Crosbie RH, Miceli MC, Spencer MJ (2009) Emerging genetic therapies to treat Duchenne muscular dystrophy. Curr Opin Neurol 22: 532 538

9. Hoffman EP, Bronson A, Levin AA, Takeda S, Yokota T, et al. (2011) Restoring dystrophin expression in duchenne muscular dystrophy muscle progress in exon skipping and stop codon read through. Am J Pathol 179: 12-22.

10. Goemans NM, Tulinius M, van den Akker JT, Burm BE, Ekhart PF, et al. (2011) Systemic administration of PRO051 in Duchenne's muscular dystrophy. N Engl J Med 364: 1513-1522.

11. Yokota T, Lu QL, Partridge T, Kobayashi M, Nakamura A, et al. (2009) Efficacy of systemic morpholinon exonskipping in Duchenne dystrophy dogs. Ann Neurol 65: 667-676.

12. Lu QL, Yokota T, Takeda S, Garcia L, Muntoni F, et al. (2011) The status of exon skipping as a therapeutic approach to duchenne muscular dystrophy. Mo Ther 19: 9-15.

13. Henricson E, Abresch R, Han JJ, Nicorici A, Goude Keller E, et al. (2012) Percent predicted 6 minute walk distance in duchenne muscular dystrophy to account for maturational influences. PLoS Curr 4.

14. Katz R (2004) Biomarkers and surrogate markers: an FDA perspective. NeuroRX 1: 189-195.

15. Arechavala-Gomeza V, Kinali M, Feng L, Brown SC, Sewry C, et al. (2010) Immunohistological intensity measurements as a tool to assess sarcolemmaassociated protein expression. Neuropathol Appl Neurobiol 36: 265-274.

16. Taylor LE, Kaminoh YJ, Rodesch CK, Flanigan KM (2012) Quantification of Dystrophin Immunofluorescence in Dystrophinopathy Muscle Specimens. Neuropathol Appl Neurobiol 38: 591-601.

17. Spitali P, Heemskerk H, Vossen RH, Ferlini A, den Dunnen JT, et al. (2010) Accurate quantification of dystrophin mRNA and exon skipping levels in duchenne muscular dystrophy. Lab Invest 90: 1396-1402.
18. Gygi SP, Rochon Y, Franza BR, Aebersold R (1999) Correlation between protein and mRNA abundance in yeast. Mol Cell Biol 19: 1720-1730.

19. Sharma N, Medikayala S, Defour A, Rayavarapu S, Brown KJ (2012) Use of quantitative membrane proteomics identifies a novel role of mitochondria in healing injured muscles. J Biol Chem 287: 30455-30467.

20. Kruger M, Moser M, Ussar S, Thievessen I, Luber CA, et al. (2008) SILAC mouse for quantitative proteomics uncovers kindlin-3 as an essential factor for red blood cell function. Cell 134: 353-364.

21. Hoffman EP, Fischbeck KH, Brown RH, Johnson M, Medori R, et al. (1988) Characterization of dystrophin in musclebiopsy specimens from patients with Duchenne's or Becker's muscular dystrophy. N Engl J Med 318: 1363-1368.

22. Jensen ON, Wilm M, Shevchenko A, Mann M (1999) Sample preparation methods for mass spectrometric peptide mapping directly from 2-DE gels. Methods Mol Biol 112: 513-530.

23. Tennyson CN, Klamut HJ, Worton RG (1995) The human dystrophin gene requires 16 hours to be transcribed and is cotranscriptionally spliced. Nat Genet 9: 184-190.

24. McClatchy DB, Yates JR III (2008) Stable Isotope Labeling of Mammals (SILAM).

25. Ardite E, Perdiguero E, Vidal B, Gutarra S, Serrano AL, et al. (2012) PAI1-regulated miR-21 defines a novel age-associated fibrogenic pathway in muscular dystrophy. J Cell Biol 196: 163-175.

26. Mendell JR, Campbell K, Rodino Klapac L, Sahenk Z, Shilling C, et al. (2010) Dystrophin immunity in Duchenne's muscular dystrophy. N Engl J Med 363: 1429-1437.

27. Patel K, Morgan J (2012) 185th ENMC International Workshop: stem/precursor cells as a therapeutic strategy for muscular dystrophies 3-5 June 2011 Naarden, The Netherlands. Neuromuscul Disord 22: 447-452.

28. Dubowitz V, Sewry CA (2007) Muscle biopsy: a practical approach. 